\title{
LiPB Battery SOC Estimation Using Extended Kalman Filter Improved with Variation of Single Dominant Parameter
}

\author{
Novie Ayub Windarko* and Jaeho Choi ${ }^{\dagger}$ \\ * Electronic Engineering Polytechnic Institute of Surabaya, Surabaya, Indonesia \\ ${ }^{\dagger}$ School of Electrical Engineering, Chungbuk National University, Chungbuk, Korea
}

\begin{abstract}
This paper proposes the State-of-charge (SOC) estimator of a LiPB Battery using the Extended Kalman Filter (EKF). EKF can work properly only with an accurate model. Therefore, the high accuracy electrical battery model for EKF state is discussed in this paper, which is focused on high-capacity LiPB batteries. The battery model is extracted from a single cell of LiPB 40Ah, 3.7V. The dynamic behavior of single cell battery is modeled using a bulk capacitance, two series RC networks, and a series resistance. The bulk capacitance voltage represents the Open Circuit Voltage (OCV) of battery and other components represent the transient response of battery voltage. The experimental results show the strong relationship between OCV and SOC without any dependency on the current rates. Therefore, EKF is proposed to work by estimating OCV, and then is converted it to SOC. $\mathrm{EKF}$ is tested with the experimental data. To increase the estimation accuracy, EKF is improved with a single dominant varying parameter of bulk capacitance which follows the SOC value. Full region of SOC test is done to verify the effectiveness of EKF algorithm. The test results show the error of estimation can be reduced up to max $5 \%$ SOC.
\end{abstract}

Key Words: Electrical model of LiPB battery, Extended Kalman Filter, Single parameter variation, SOC estimation

\section{INTRODUCTION}

Existing energy storage systems are including compressedair energy storage (CAES), flywheels energy storage, pumpedstorage hydroelectricity, super conducting magnetic energy storage (SMES), ultracapacitors and batteries. Each of those energy storages has its own advantages and disadvantages. Among those energy storage systems, batteries have advantages of fast response speed, high ramp rates, easily sited, modular, and good energy efficiency [1].

Batteries as the energy storage equipment can be operated for several functions, such as to increase the reliability of supply [2], power quality [3], the efficiency of operation [4], etc. Those functions can be found in Microgrid system which comprises the uncontrollable energy sources such as wind turbine, PV power, etc. Further, those functions of battery need a battery management system (BMS) which supports the battery operation by several tasks. For example, it can prevent the battery from entering into overcharging- or overdischargingcondition and give the information of residual energy to users [5]. Mainly, BMS needs the State-of-Charge (SOC) estimator

Manuscript received Sep. 6, 2011; revised Nov. 29, 2011

Recommended for publication by Associate Editor Jun-Keun Ji.

$\dagger$ Corresponding Author: choi@chungbuk.ac.kr

Tel: +82-43-261-2425, Chungbuk National University

* Electronic Engineering Polytechnic Institute of Surabaya, Indonesia

${ }^{\dagger *}$ School of Electrical Eng., Chungbuk National University, Korea to do the tasks.

The methods of SOC estimation can be categorized by the direct measurement based method or the battery model based one. Coulomb counting or current integration is one of the examples of direct measurement as the most popular method by its simplicity. However, it leads to high errors due to the accumulation of error integration in current measurement. And Kalman Filter (KF) or Extended Kalman Filter (EKF) can be one of the representative battery model based methods. KF is introduced to estimate SOC with its several advantages. KF can optimally (minimum variance) estimate the states affected by the broadband noise contained within the system bandwidth, i.e., that cannot be filtered out using classical techniques, and enable the empirical tradeoffs between modeling errors and the influence of noise [6]. While KF is working in linear systems, EKF can estimate properly in nonlinear system [7].

To develop a battery model, many previous studies have been done. Several models have been reported using electrochemical base [8], [9]. Other researchers have proposed electrical battery models consisting of resistances and capacitances [10], [11]. The electrical model is preferred by the electrical engineers due to the easiness for them to understand. Furthermore, mostly the battery model is developed based on low capacity type for electronic appliances. Contrarily, battery application for high power application uses high capacity type. 


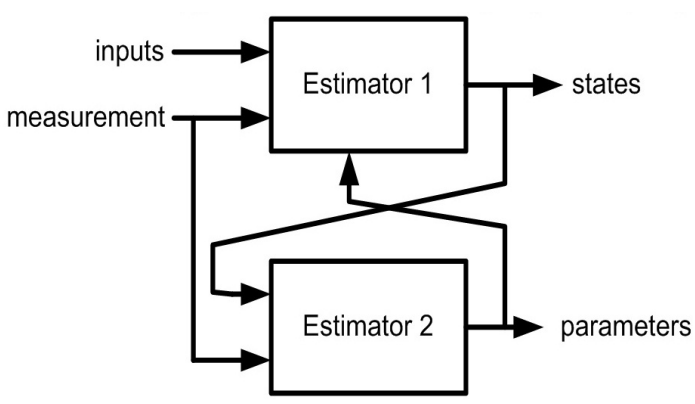

Fig. 1. Dual estimator to obtain varying parameters.

Several researchers have developed a battery model of high capacity type [11], [12]. The model can perform in high accuracy. It is reported that the model can represent the battery voltage response with error less than $12 \mathrm{mV}$ of single cell $1.2 \mathrm{~V}$ NiMH type [11] and less than $5 \mathrm{mV}$ of single cell $3.7 \mathrm{~V} \mathrm{LiPB}$ type [12].

In papers [6] and [7], KF and EKF are used to estimate SOC, respectively. In those papers, a lead acid battery is used for experiment. The characteristic of this battery is simpler than that of Lithium type. The researchers have adopted a battery model consisting of two parallel series RC circuits and a series resistance. The results show that estimated OCV and true OCV has error higher than $0.5 \mathrm{~V}$. This error may be caused by the mismatching in battery modeling.

KF based SOC estimation method of batteries in the energy systems for PV application has been proposed [13]. The researchers used a first order of electrical model for KF states. It was reported that the error could be kept below $10 \%$ for data of a $12 \mathrm{~V} 125 \mathrm{Ah}$ flooded a lead-acid battery cycled on a typical $\mathrm{PV}$ regime over a period of 2.5 years.

EKF has been adopted to estimate SOC for battery packs [14]. The battery pack for an experiment is Lithium ion type of $330 \mathrm{~V}, 15 \mathrm{Ah}$. The underlying dynamic behavior of the battery pack was described by a model comprising of two capacitances and three resistances. The maximum error was less than $2 \mathrm{~V}$. Some specific current tests were applied to validate the proposed method. To verify the performance of EKF, the results were compared with those of coulomb counting. The results showed that the methodologies were able to perform properly to estimate SOC of the battery pack. The test was done in the narrow region of SOC. Results in the tests showed that the maximum error was less than $3 \%$.

Several authors have used Randle's battery model consisted of series resistance, single RC network, and bulk capacitance [15]. The model parameter was based on rest condition instead of load condition. But, the load condition is much more important to observe the parameters than in the rest condition. For example, OCV during the load condition changes following the change in SOC, while in rest condition it does not change. In paper [16], a coulomb counting based EKF has been proposed for SOC estimation. Randle's battery model has been adopted with the single RC network. However the results were shown that the SOC estimation error tended to be increased, and it might be caused by a coulomb counting algorithm adopted in EKF.

The time varying parameters for system to increase the

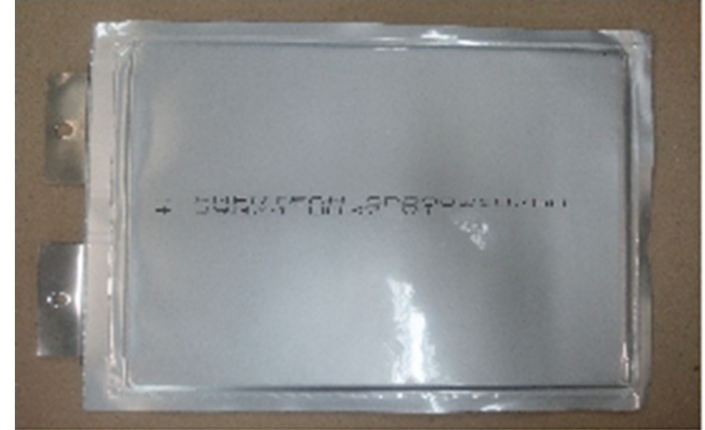

Fig. 2. Li-polymer Enertech, SPB90210260V1, prismatic single cell module, $3.7 \mathrm{~V}$ and $40 \mathrm{Ah}$.

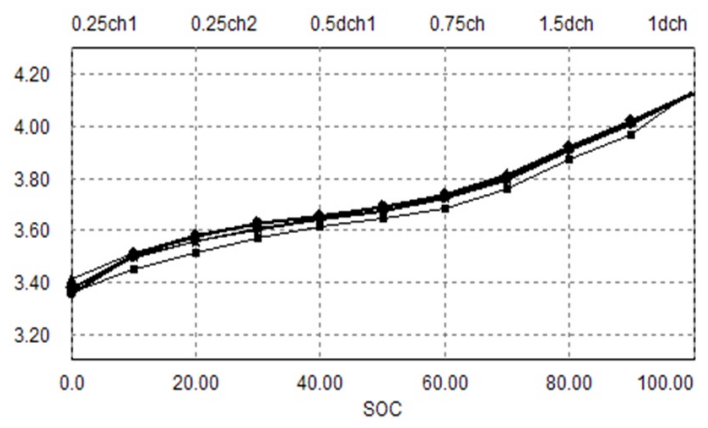

Fig. 3. OCV of LiPB battery in charging and discharging.

estimation accuracy have been proposed by some researchers. They have proposed an additional estimator to perform the varying parameters. Dual EKF was studied to estimate the states and parameters as shown in Fig. 1. The first EKF was employed to estimate the state of battery and the second one was done to estimate the parameters of battery model [17]. Furthermore, a dual sliding mode observer was proposed to estimate the states and parameters. The first observer was a fast time-varying observer which estimated the parameters including SOC. The second one was a slow time-varying observer to estimate the state of health [18]. However, applying dual observers might increase the calculation time of the controller/processor.

This paper proposes the application of EKF to estimate SOC. EKF is proposed to be worked by estimating OCV, and then it is converted to SOC. This principle is based on the fact that OCV and SOC have the strong relation and have no dependency of current rates [19], [20]. The state space equation of the battery in EKF is used a high accuracy electrical model based on paper [12]. This model consists of a bulk capacitance, double RC networks and a series resistance. The performance of EKF is improved with a single dominant varying parameter. Based on the knowledge in battery modeling, it is found that the bulk capacitance influences the battery voltage dominantly. Therefore, the bulk capacitance is chosen for the varying parameter by following the SOC value. Moreover, EKF algorithm is tested in full region of $\mathrm{SOC}$ and various current rates.

\section{BATTERY ELECTRICAL MODEL}

Battery for experiment is LiPB Enertech, SPB90210260V1, prismatic single cell module, $3.7 \mathrm{~V}$ and $40 \mathrm{Ah}$. It is shown in Fig. 2. This type of battery has merit of high capacity which 


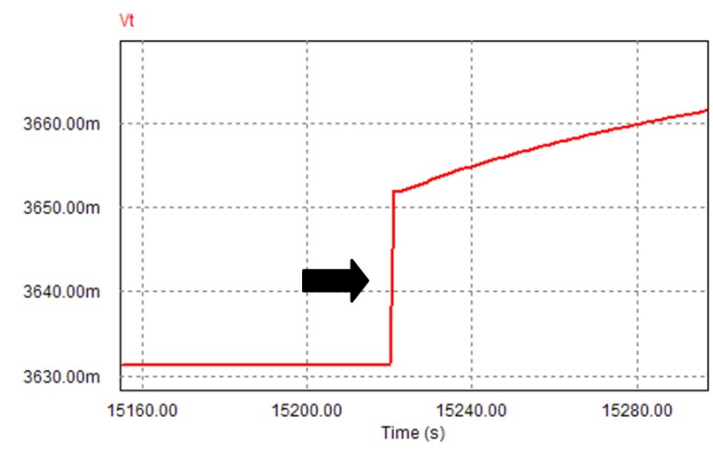

Fig. 4. Response of internal resistance to pulse current.

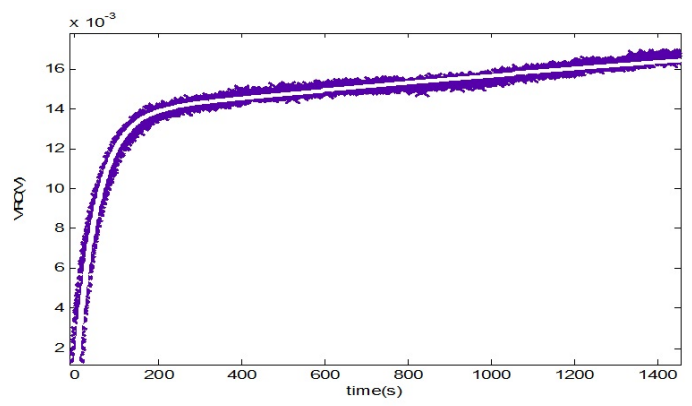

(a)

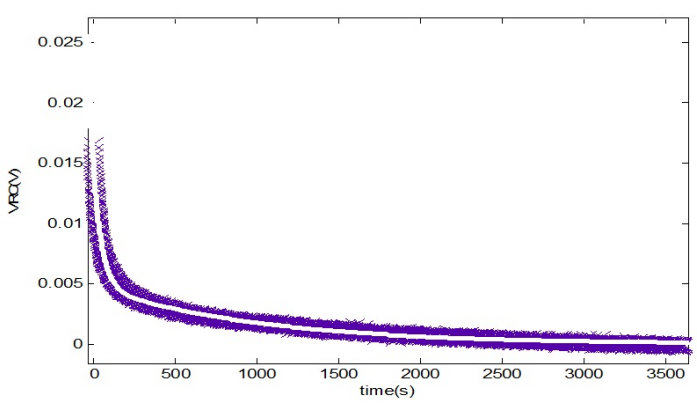

(b)

Fig. 5. Curve fitting of RC networks voltage, $\mathrm{V}_{R C}$, from LiPb battery: (a) loaded and (b) rest time.

is suitable for Renewable energy application. The experiment is performed at $30^{\circ} \mathrm{C}$. During the experiments, the increasing of battery temperature can be negligible. The MACCOR automatic battery tester is used to test the battery. The battery tester can be programmed to produce the pulse current for charging and discharging processes. This tester could also monitor the battery conditions including temperature, terminal voltage, and current. Every data are recorded at every second.

The OCV measurement is done by applying constant pulse current which is equal to $10 \%$ SOC. It is done for both of charging and discharging modes. The test result in charging is by $0.25 \mathrm{C}$ and $0.75 \mathrm{C}$, and in discharging is by $0.5 \mathrm{C}, 1 \mathrm{C}$ and $2 \mathrm{C}$. Figure 3 shows $\mathrm{OCV}$ in charging and discharging. These results confirm the conclusion on previous reports: OCV curve is not influenced by the charging and discharging rates and are similar between charging and discharging [19], [20]. However this conclusion should be limited only for Lithium type of battery. It cannot be guaranteed that this conclusion is correct for other types. With the similarity of charging and discharging $\mathrm{OCV}$, the minor loop and the major loop in OCV

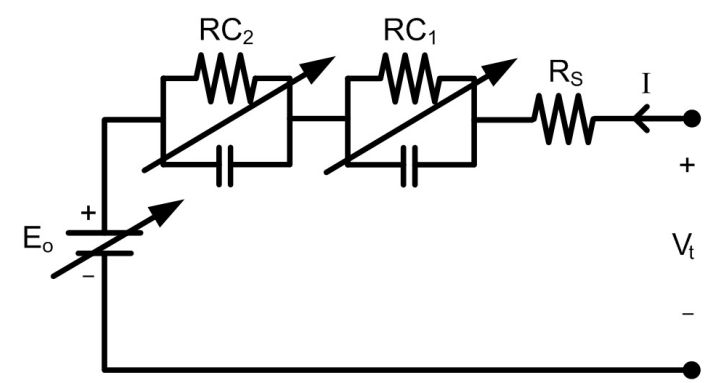

Fig. 6. Proposed RC networks for LiPB battery.

can be neglected. The fitting model for OCV can be done simply by MATLAB.

The internal resistance of $R_{s}$ can be identified with its linear response just after current changed in terminal voltage. Figure 4 shows this response with an arrow indicator. Resistance Rs can be calculated from the terminal voltage response just before and after the current changed. The resistance can be calculated by:

$$
R_{s}=\left|\frac{V_{t a}-V_{t b}}{I}\right|
$$

which $V_{t a}$ is the voltage just after charging and $V_{t b}$ is the voltage just before charging and $I$ is the battery current. Note that, the resistance value must be positive. Therefore the absolute function kept $R_{S}$ value in positive during discharge mode which $\left(V_{t a}-V_{t b}\right)$ is negative.

The RC networks can be identified by exponential response to pulse current. The exponential response is occurred during loaded and rest conditions. However, it should be checked the order of the exponential response. Figure 5 shows the response of RC networks and its curve fitting. MATLAB is used to fit the curve and to calculate the exponential order and time constants. The curve fitting results shows that the RC network voltage is similar to second-order in both of loaded and rest conditions. Even though the order is same but the time constant is different. Concerning to these characteristics, the secondorder RC network is proposed. It should be note that, the time constant of RC network is also SOC value dependent. Consider to those characteristics, the proposed circuit should be dependent on SOC value. The proposed electrical model has arrow signs to indicate its dependency to SOC value which is shown in Fig. 6. During the loaded condition, the electrical model in Fig. 6 can be expressed as:

$V_{t}(t)=I * R_{S}+I * R_{1} *\left(1-e^{-t / R_{1} C_{1}}\right)+I * R_{2} *\left(1-e^{-t / R_{2} C_{2}}\right)+E_{o}$

where $V_{t}$ is terminal voltage, $I$ is current, $R_{1}$ and $R_{2}$ and $C_{1}$ and $C_{2}$ are resistances and capacitances in RC network 1 and 2, respectively. During rest time, the electrical model in Fig. 6 can be expressed as:

$$
V_{t}(t)=V_{R 1 C 1} *\left(1-e^{-t / R_{1} C_{1}}\right)+V_{R 2 C 2} *\left(1-e^{-t / R_{2} C_{2}}\right)+E_{o}
$$

where $V_{R 1 C 1}$ and $V_{R 2 C 2}$ are voltages across $R_{1}$ or $C_{1}$ and across $R_{2}$ or $C_{2}$ respectively. $V_{R C}$ value is determined just after the current turn to zero.

The results of modeling applied in simulation are shown in Figs. 7 and 8. For the verification of modeling accuracy, the simulation results are compared with the experiment data. 


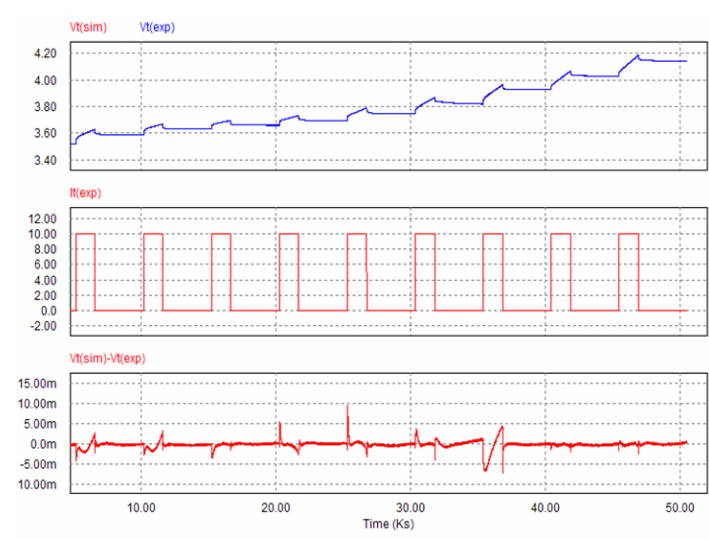

Fig. 7. LiPB battery charging operation: (top) Simulation result and experiment data, $V_{t}(\mathrm{~V})$, (middle) pulse current $(\mathrm{A})$, (bottom) error of simulation (V).

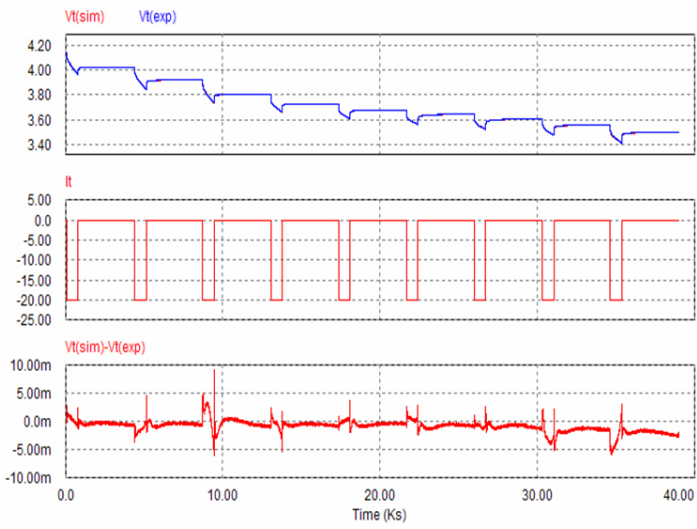

Fig. 8. LiPB battery disharging operation: (top) Simulation result and experiment data, $V_{t}(\mathrm{~V})$, (middle) pulse current $(\mathrm{A})$, (bottom) error of simulation (V).
The charging operation is shown in Fig. 7. The battery is charged with $0.25 \mathrm{C}(10 \mathrm{~A})$ until the end-of-charge voltage, $4.2 \mathrm{~V}$. Further, the discharging operation is done with $0.5 \mathrm{C}$ (20A). The battery is discharged to the end-of-discharge voltage. The error between simulation and experiment data for charging operation are shown. The discharging operation has similar results as shown in Fig. 8. In several points, the error shows similar spikes produced by one step iteration lagging of simulator relative to the experiments data. Nevertheless, the maximum absolute average error of modeling can be reduced up to $5.5 \mathrm{mV}$. While the absolute error averaged by the iteration time is close to zero.

\section{Kalman Filter And Extended Kalman Filter THEORY}

To illustrate this theory, consider the linear discrete time system in Fig. 9. The determined input to the system is $i_{k}$, and the output or response of the systems is $y_{k} . A_{k}$, $B_{k}$, and $C_{k}$ are matrices, and $z^{-1}$ is the unit-delay operator which represents the system in the mathematical equation. The system is distorted by two stochastic noises: process noise $w_{k}$ and measurement noise $v_{k}$. It is assumed that the noises are white noise. The output $y_{k}$ is measured by some devices. The result of measurement is $m_{k}$ distorted by noise $v_{k}$. Then, $x_{k}$ is the system state. Commonly the state $x_{k}$ is unknown and may not be measured directly. The value of state $x_{k}$ can be estimated by Kalman filter through the model. To estimate the state value, the $y_{k}$ can be used to correct the prediction. Note that, the measured value of $y_{k}$ is $m_{k}$ which contains measurement noise $v_{k}$.

The system in Fig. 9 can be expressed mathematically into two equations. The first describes the state equation expressed as:

$$
x_{k+1}=A_{k} x_{k}+B_{k} i_{k}+w_{k} .
$$

It is assumed that $w_{k}$ is additive, white and Gaussian, with zero mean and covariance matrix. The process noise $w_{k}$ can be expressed as:

$$
E\left[w_{n} w_{k}^{T}\right]=\left\{\begin{array}{c}
\Sigma_{w}, n=k \\
0, n \neq k
\end{array}\right.
$$

The second describes the measurement equation expressed as:

$$
m_{k}=y_{k}+v_{k}
$$

And, replaces the $y_{k}$, then Eq. 6 becomes:

$$
m_{k}=C_{k} x_{k}+v_{k} .
$$

Equation 7 shows that the output of the system $y_{k}$ with discrete-time index $k$ is a linear combination of states $x_{k}$ and matrices $C_{k}$. Then, the measured output of $m_{k}$ contains the measurement noise of $v_{k}$. Kalman filter theory assumes that the measurement noise $v_{k}$ is additive, white and Gaussian with zero mean and covariance matrix. It is similar to the process noise. The measurement noise can be expressed as:

$$
E\left[v_{n} v_{k}^{T}\right]=\left\{\begin{array}{c}
\Sigma_{v}, n=k \\
0, n \neq k .
\end{array}\right.
$$


The Kalman filter aim is to use the entire observed data of input $\left\{i_{1}, i_{2}, \ldots i_{k}\right\}$ and measured output $\left\{m_{1}, m_{2}, \ldots m_{k}\right\}$ to find the minimum mean squared error estimate $\hat{x}_{k}$ of the true state $x_{k}$.

The main algorithm of the solution is a set of recursive relationships that involve both of estimation of the state itself and also the covariance matrix of $\Sigma_{e, k}$ of the state estimate error of $x_{k}-\hat{x}_{k}$. The covariance matrix indicates the uncertainty of the estimation, and may be used to generate error bounds on the estimation. Large value of $\Sigma_{e, k}$ indicates a high level of uncertainty of the estimation, while a small value of $\Sigma_{e, k}$ indicates the certainty of the estimation.

The illustration of the Kalman Filter algorithm is shown in Fig. 10. The Kalman filter is initialized with the best available information on the state and error covariance. The initialized value of state estimation and error covariance are expressed as:

$$
\begin{aligned}
& \hat{x}_{0}=E\left[\hat{x}_{0}\right] \\
& \sum_{x, 0}(+)=E\left[\left(\hat{x}_{0}-E\left[x_{0}\right]\right)\left(x_{0}-E\left[x_{0}\right]\right)^{T}\right] .
\end{aligned}
$$

The initialization is only to support the initial estimation, and then the KF will seek the true values by the iteration steps. Following the initialization, KF repeats two steps. Firstly, it predicts the value of the next state, system output, and error covariance. The predicted state and error covariance estimates before the measurement at time index $k$ are denoted $\hat{x}_{k}(-)$ and $\Sigma_{e, k}(-)$, respectively. These calculations are done in Time Update (Prediction) box in Fig. 10. Secondly, using a measurement of the system output, it corrects the present state estimate. The corrected estimates and error covariance following the measurement are denoted $\hat{x}_{k}(+)$ and $\Sigma_{e, k}(+)$. These calculations are done in Measurement Update (Correction) box in Fig. 10. The prediction step is accomplished by propagating the system input $i_{k}$ through the system dynamics. The prediction for the system state is expressed as:

$$
x_{k+1}(-)=A_{k} \hat{x}_{k}(+)+B_{k} i_{k} .
$$

The uncertainty of prediction is also updated and expressed as:

$$
\sum_{e, k+1}(-)=A_{k} \sum_{e, k}(+) A_{k}^{T}+\sum_{w}
$$

If the system is stable, the first term is contractive and it can reduce the uncertainty. The process noise $\Sigma_{w}$ term always increases the uncertainty. The state correction step is expressed as:

$$
x_{k}(+)=\hat{x}_{k}(-)+L_{k}\left[m_{k}-C_{k} \hat{x}_{k}(-)\right] .
$$

That is, the new state estimate equals the value of the predicted state estimate plus a weighted correction factor. The term in the square brackets is equal to the value of the measured terminal voltage minus the predicted terminal voltage. This term may be non-zero due to the measurement noise, an incorrect state estimate $\hat{x}_{k}(-)$, or an inaccurate battery model. It represents the "new information" in the measurement, and is often called the "innovation" process for this reason. If the innovation is large, the state update tends to be large. If the innovation is small, the state update tends to be small. The innovation is weighted by the Kalman gain $L_{k}$ is expressed as:

$$
L_{k}=\sum_{e, k}(-) C_{k}^{T}\left[C_{k} \sum_{e, k}(-) C_{k}^{T}+\sum_{v}\right]^{-1} .
$$

If the present state estimate is very uncertain, $\Sigma_{e, k}$ is large and $L_{k}$ is large, forcing a large update. If the present state estimate is certain, $L_{k}$ is small, and the state-estimate update is small. Also, if sensor noise is large, $\Sigma_{v}$ is large, causing $L_{k}$ to be small and the update to be small. The covariance correction step is expressed as:

$$
\sum_{e, k}(+)=\sum_{e, k}(-)-L_{k} C_{k} \sum_{e, k}(-) .
$$

The state certainty always increases due to the new information provided by the measurement. The Kalman filter is the optimum state estimator for a linear system with the assumptions as described. If the system is nonlinear, then we may use a linearization process at every time step to approximate the nonlinear system with a linear time-varying (LTV) system. This LTV system is then used in the Kalman filter, resulting in an extended Kalman filter (EKF) on the true nonlinear system. Although EKF is not necessarily optimal, it often works very well. The nonlinear system may be modeled as:

$$
\begin{gathered}
x_{k+1}=f\left(x_{k}, i_{k}\right)+w_{k} \\
m_{k}=g\left(x_{k}, i_{k}\right)+v_{k} .
\end{gathered}
$$

As before, $w_{k}$ and $v_{k}$ are zero mean white Gaussian stochastic processes with covariance matrices $\Sigma_{w}$ and $\Sigma_{v}$, respectively. Now, $f\left(x_{k}, i_{k}\right)$ is a nonlinear transition matrix function and $g\left(x_{k}, i_{k}\right)$ is a nonlinear measurement matrix.

At each time step, $\mathrm{f}\left(x_{k}, i_{k}\right)$ and $g\left(x_{k}, i_{k}\right)$ are linearized by a Taylor-series expansion.

$$
f\left(x_{k}, i_{k}\right) \approx f\left(\hat{x}_{k}, i_{k}\right)+\left.\frac{\partial f\left(x_{k}, i_{k}\right)}{\partial x}\right|_{x_{k}=\hat{x}_{k}}\left(x_{k}-\hat{x}_{k}\right)
$$

where

$$
\begin{gathered}
\left.\frac{\partial f\left(x_{k}, i_{k}\right)}{\partial x}\right|_{x_{k}=\hat{x}_{k}}=A_{k} \\
g\left(x_{k}, i_{k}\right) \approx g\left(\hat{x}_{k}, i_{k}\right)+\left.\frac{\partial g\left(x_{k}, i_{k}\right)}{\partial x}\right|_{x_{k}=\hat{x}_{k}}\left(x_{k}-\hat{x}_{k}\right) \\
\left.\frac{\partial g\left(x_{k}, i_{k}\right)}{\partial x}\right|_{x_{k}=\hat{x}_{k}}=C_{k}
\end{gathered}
$$

So, it can be described as the linearized system:

$$
\begin{gathered}
x_{k+1} \approx A_{k} x_{k}+\left[f\left(\hat{x}_{k}, i_{k}\right)-A_{k} \hat{x}_{k}\right]+w_{k} \\
m_{k} \approx C_{k} x_{k}+\left[f\left(\hat{x}_{k}, i_{k}\right)-C_{k} \hat{x}_{k}\right]+v_{k} .
\end{gathered}
$$




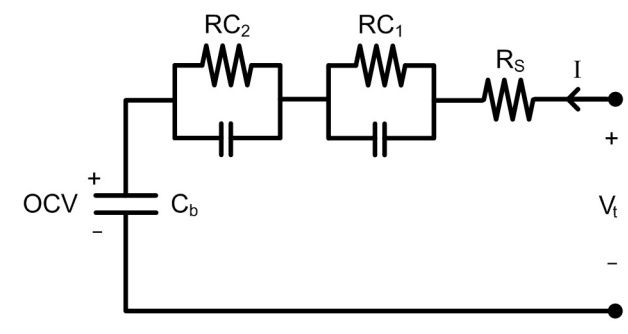

Fig. 11. Battery model with RC network.
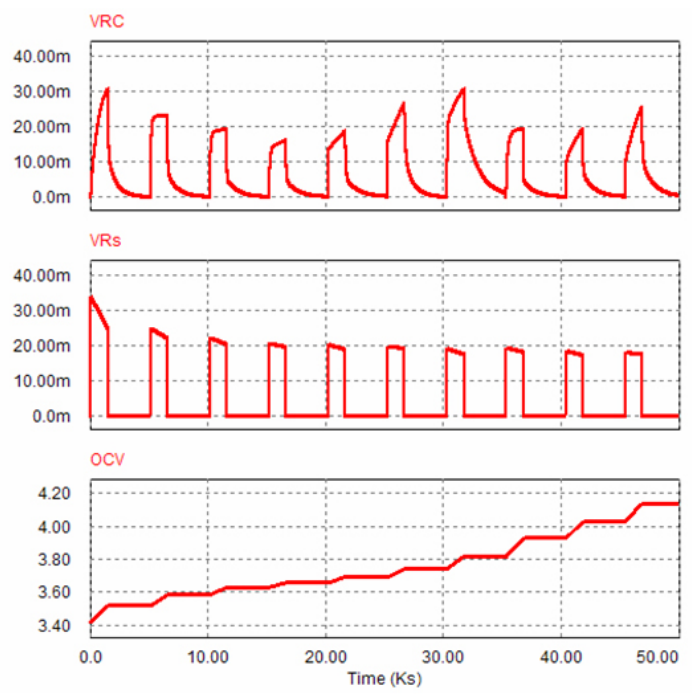

Fig. 12. Voltage of RC network (top), internal resistance (middle), and OCV (bottom).

\section{EKF BASED ON ElECTRICAL BATTERY MODEL}

This section explains the adopted electrical model for the Extended Kalman Filter. The adopted model is shown in Fig. 11 which based on electrical model in Fig. 5. The model consists of bulk capacitance to represent OCV, double $\mathrm{RC}$ network to represent the transient response and internal resistance to represent the linear response of terminal voltage. Consider to Fig. 11, the current equation for RC network can be expressed as:

$$
I=I_{c 1}+I_{r 1}
$$

which $I_{c 1} / I_{r 1}$ is current flows to capacitance/resistance of $\mathrm{R}_{1}$ $\mathrm{C}_{1}$ network respectively. The current flows to capacitance can be expressed as:

$$
I_{c 1}=C_{1} \frac{d V c_{1}}{d t}
$$

The current flow to resistance can be expressed as:

$$
I_{r 1}=\frac{V c_{1}}{R_{1}}
$$

Then, substituting Eqs. 24 and 25 to Eq. 23, we can get:

$$
I=C_{1} \frac{d V c_{1}}{d t}+\frac{V c_{1}}{R_{1}}
$$

The state equation for the EKF should be in differential form to linearize the nonlinear system. Therefore, the equation should be formed in differential. Adopting Eq. 26, the differential voltage across capacitance in $R_{1}-C_{1}$ circuit is expressed as:

$$
\frac{d V c_{1}}{d t}=-\frac{V c_{1}}{R_{1} C_{1}}+\frac{I}{C_{1}}
$$

The differential voltage across capacitance in $\mathrm{R}_{2}-\mathrm{C}_{2}$ circuit is same as in $\mathrm{R}_{1}-\mathrm{C}_{1}$ :

$$
\frac{d V c_{2}}{d t}=-\frac{V c_{2}}{R_{2} C_{2}}+\frac{I}{C_{2}} .
$$

The differential voltage equation in bulk capacitance is expressed as:

$$
d V c_{b}=\frac{I_{c}}{C_{b}} d t .
$$

Finally, to show the relation of all internal components voltage, the voltage equation for battery is expressed as:

$$
V_{t}=V_{c b}+V_{c 1}+V_{c 2}+V_{R s} .
$$

$\mathrm{EKF}$ is the optimum state estimator for nonlinear system worked by recursion. To calculate in recursion, EKF is formed in the discrete-time state space equations as shown in Eqs. 15 and 16. Therefore the equation should be transformed into discrete form. The RC network voltage equation can be expressed in a discrete form as:

$$
V c_{n(k+1)}=V c_{n(k)}\left(1-\frac{T}{R_{n} C_{n}}\right)+\frac{T . I_{k}}{C_{n}}
$$

where $V_{c n(k+1)}$ and $V_{c n(k)}$ are the next and the present voltage values of capacitance $C_{n}$, respectively, where $T$ is the sampling time of digital simulator, $I_{k}$ is the present value of battery current, and $k$ is iteration.

The bulk capacitance voltage equation can be expressed in discrete form as:

$$
V c_{b(k+1)}=V c_{b(k)}+\frac{I_{c} \cdot T}{C_{b}}
$$

where $V_{c b(k+1)}$ and $V_{c b(k)}$ are the next and the present voltage values of capacitance $C_{b}$, respectively .

By following the form in EKF, the state equations for the system are $x_{1}=V_{c 1}, x_{2}=V_{c 2}$ and $x_{3}=V_{c b}$. All of the states in battery cannot be measured directly. While the input $I$ and measured output $V_{t}$ can be measured directly. The state space equation of the battery and measurement equation are expressed as:

$$
\begin{aligned}
& {\left[\begin{array}{c}
x_{1(k+1)} \\
x_{2(k+1)} \\
x_{3(k+1)}
\end{array}\right]=\left[\begin{array}{c}
V_{c 1(k+1)} \\
V_{c 2(k+1)} \\
V_{c b(k+1)}
\end{array}\right]=} \\
& {\left[\begin{array}{ccc}
1-\frac{T}{R_{1} C_{1}} & 0 & 0 \\
0 & 1-\frac{T}{R_{2} C_{2}} & 0 \\
0 & 0 & 1
\end{array}\right]\left[\begin{array}{l}
x_{1(k)} \\
x_{2(k)} \\
x_{3(k)}
\end{array}\right]+\left[\begin{array}{c}
\frac{T}{C_{1}} \\
\frac{T}{C_{2}} \\
\frac{T}{C_{b}}
\end{array}\right] I_{k}} \\
& m_{k}=V_{t(k)}=x_{1(k)}+x_{2(k)}+x_{3(k)}+i_{k} \cdot R_{s} .
\end{aligned}
$$

EKF needs an accurate model to perform the estimation works well. For this purpose, the first EKF algorithm is proposed as shown in Fig. 13. It shows that EKF uses the battery current as input and the battery voltage for measured output. The main task of EKF algorithm is to estimate OCV. Then OCV is converted to SOC by the look-up table. To verify EKF performance, the battery is tested using the pulse current load. The pulse current is $1 \mathrm{C}$ (40A) charging. The battery voltage and current is recorded by MACCOR battery tester. The recorded data was used to test the performance of EKF 
algorithm. The initial value of OCV estimation for EKF is $4 \mathrm{~V}$. Figure 14 shows the performance of EKF. Figure 14(a) shows the measured battery current, and Fig. 14(b) shows that the terminal voltage response of battery and its estimation. Figure 14(b) shows the estimated terminal voltage which is very similar with measured data. It is verified that terminal voltage can be estimated well by EKF. Figure 14(c) shows EKF uses the initial estimation of OCV at $4 \mathrm{~V}$. EKF tries to track OCV at the beginning of steps. After then, EKF works to reduce the error by recursion and starts to get the correct value at around $10 \%$ SOC. But EKF cannot track well the OCV in the region of high SOC and finally EKF starts to lose the tracking ability at $70 \%$ SOC. The SOC estimation error tends to be higher as the increase of SOC. At $100 \%$ SOC, the error of estimation reaches around $20 \%$ SOC. These results show that even the estimation for the terminal voltage is good, but the estimation for OCV is unsatisfying and need to improve it. The large error in estimation may be caused by the inaccuracy of battery model. Therefore, an improvement of battery modeling is necessary to reduce the error of estimation.

Some researchers have proposed dual estimator to perform a high accuracy model and the main estimator itself. However this solution needs long calculation and time consuming. Therefore, considering the problems and limitations, it is proposed a single varying parameter. The single varying parameter is chosen as the dominant one among all of the model parameters. By choosing a dominant one, it is expected that it does not impose burden on EKF to reduce the error. Therefore the estimation can be kept in high accuracy. Based on Fig. 12, it can be recognized that OCV is the most dominant comparing with other voltage parameters. Under the open circuit condition, OCV will be much more dominant. Simply, $\mathrm{OCV}$ is the most dominant value and then chosen as a single varying parameter. However, OCV cannot be implemented directly as a single varying parameter in EKF. Consider to Fig. $11, \mathrm{OCV}$ is represented by the voltage of bulk capacitance, $C_{b}$. Therefore the single varying parameter in EKF is represented by bulk capacitance, $C_{b}$.

The illustration of an improved EKF algorithm is shown in Fig. 15. It shows that EKF uses the battery current and voltage as the inputs to estimate the SOC. By using both battery current and voltage, this algorithm can reduce the dependency on the current sensor. Therefore, the current measurement error cannot influence directly to the estimation accuracy. The current input is used to estimate the voltages of RC networks and OCV. Then OCV is converted to SOC by using the piecewise continuous linear look-up table as shown in Fig. 16. In case of look-up table, $x$ is OCV and $y$ is SOC. The look up table can calculate any points among the sampled data. Any points between the sampled data can be calculated by:

$$
y_{n}=y_{1}+\frac{\left(x_{n}-x_{1}\right) \cdot\left(y_{2}-y_{1}\right)}{x_{2}-x_{1}} .
$$

Error propagation is used to account the process noise which deteriorates the estimation accuracy. The estimated battery voltage is compared by the measured battery voltage after weighting by the Kalman gain calculated as the error propagation, and then the state estimation and the estimation

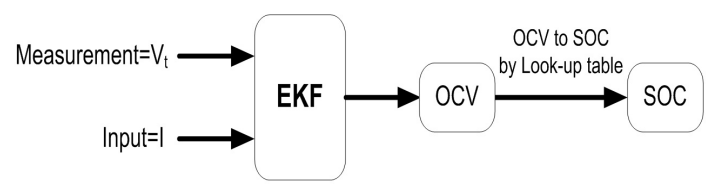

Fig. 13. EKF with constant parameters.

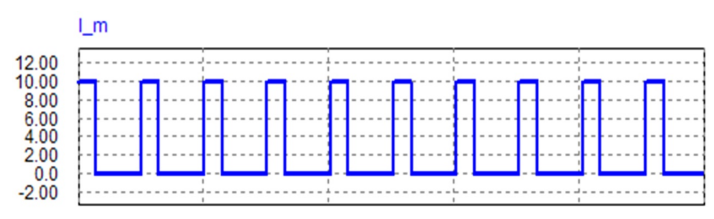

(a)

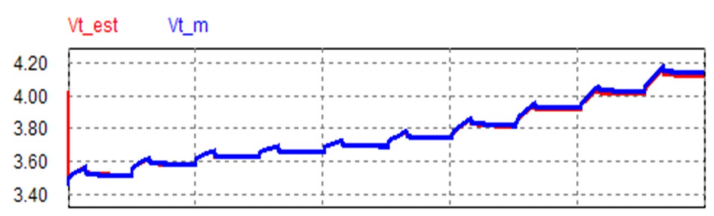

(b)

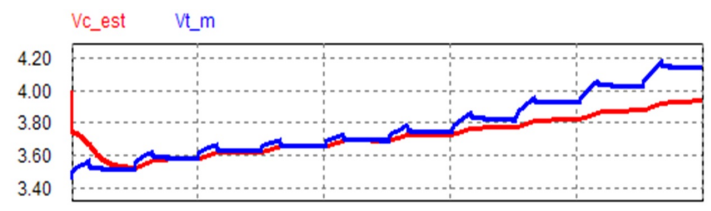

(c)

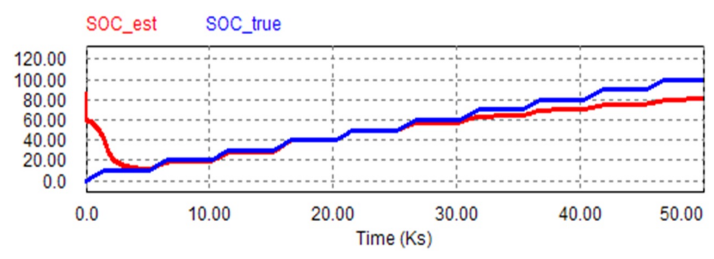

(d)

Fig. 14. Experimental data and estimation value from EKF. (a) battery current (A), (b) estimation and measured voltage (V), (c) estimation OCV and battery voltage (V), (d) SOC estimation and true value (\%SOC).

error will be updated. If the present state estimate is certain, the Kalman gain is small and the error update is also small.

Though it has been used the accurate battery model, the accumulated estimation error is increased due to the error of the battery model. To reduce the estimation error, it has been used in this work to modify the battery model with single varying parameter of bulk capacitance, $C_{b}$. To calculate the bulk capacitance, two points of SOC-OCV are used. Then from the following Eq. 36, the bulk capacitance value can be calculated by:

$$
C_{b}=\frac{S O C_{2}-S O C_{1}}{V_{2}-V_{1}}=\frac{\int I d t}{V_{2}-V_{1}} .
$$

The difference value of SOC is converted into Ah represented by Idt. The results of this calculation are shown in Fig. 17. The value has the range of around $100 \mathrm{kF}$ to $500 \mathrm{kF}$. The capacitance has the peak value at 30\%SOC. The capacitance has high value means that the voltage changes in the small range when the battery charges with the same amount of energy. 


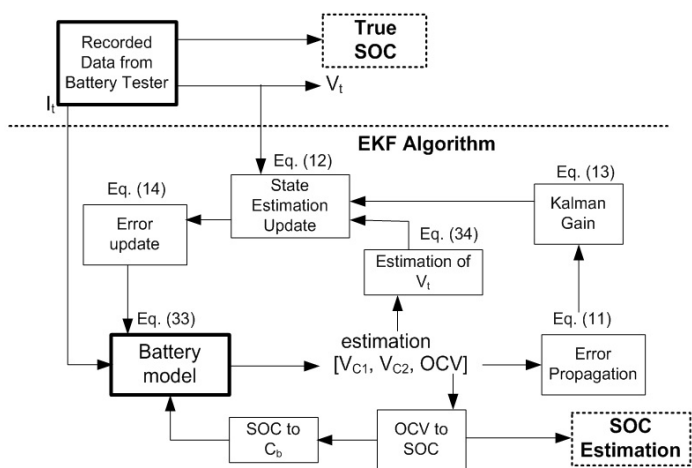

Fig. 15. EKF algorithm with single dominant varying parameter of bulk capacitance.

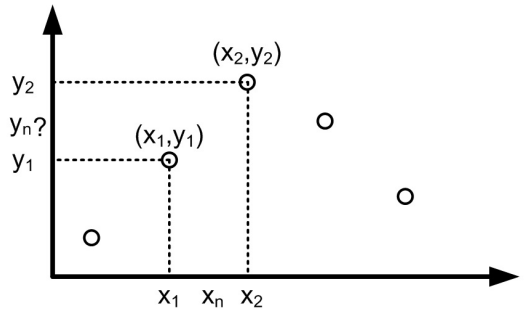

Fig. 16. Principle operation of look up table.

\section{RESULTS}

The battery is tested using a pulse current load. The battery voltage and current are recorded by the MACCOR battery tester. The recorded data are used to test the performance of EKF algorithm. The preliminary test is done by applying pulse current of $1 \mathrm{C}(40 \mathrm{~A})$ in charging operation. Figure 18 shows the test results of EKF. The figure shows that at the beginning of processes, it can estimate the battery voltage very well. Further, at the beginning of processes EKF is tried to track the true value of OCV very hard, but it can track well at around 4200 s at last. This condition can be accepted theoretically. However in real application, this condition may disturb the performance of estimator. Therefore it should be adopted in suitable application. For example, microgrid application can adopt this algorithm without any concerns. Just after the battery is installed in system, it will never disconnect from the system except under maintenance. Therefore the hard tracking is only occurred at the first time operation of battery, and then it can estimate well.

To verify the performance of EKF in more realistic conditions, it is tested by cycles of charging and discharging with various currents. The current rates are various from $0.2 \mathrm{C}$ to $2 \mathrm{C}$. Figures 19 to 20 show the verification of EKF performance by various current rate tests. It verifies that the tracking is working well with various current rates. In those figures, it is shown that the error of SOC estimation is reduced up to $5 \%$ SOC over the all SOC region.

\section{CONCLUSIONS}

To estimate the battery SOC, EKF is proposed works based on electrical battery model. The electrical battery model consists of internal resistance, double RC network and bulk capacitance. The voltage of a bulk capacitance represents OCV. SOC can be estimated by converting OCV using SOCOCV relationship. However, EKF may work improperly if the

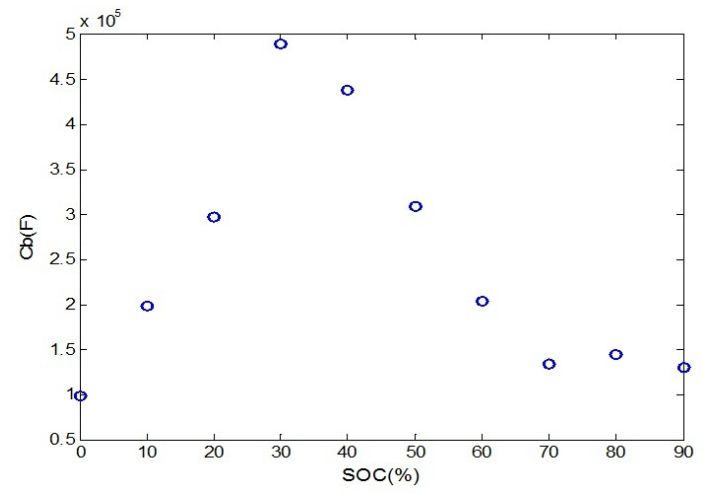

Fig. 17. Bulk capacitance value following SOC.

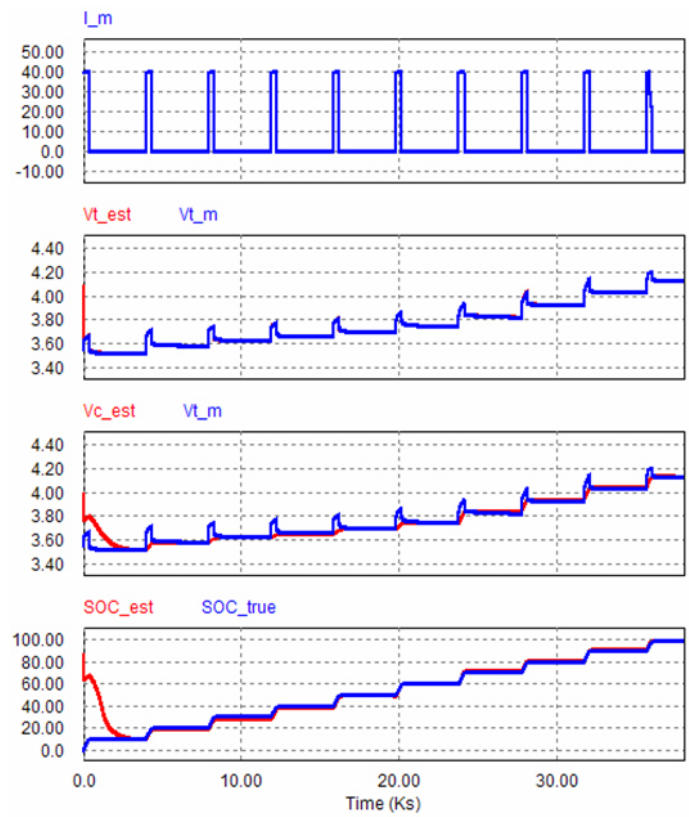

Fig. 18. Experimental data and estimation value from EKF with single dominant varying parameter. (a) battery current (A), (b) estimation and measured voltage (V), (c) estimation OCV and battery voltage (V), (d) SOC estimation and true value (\%SOC).

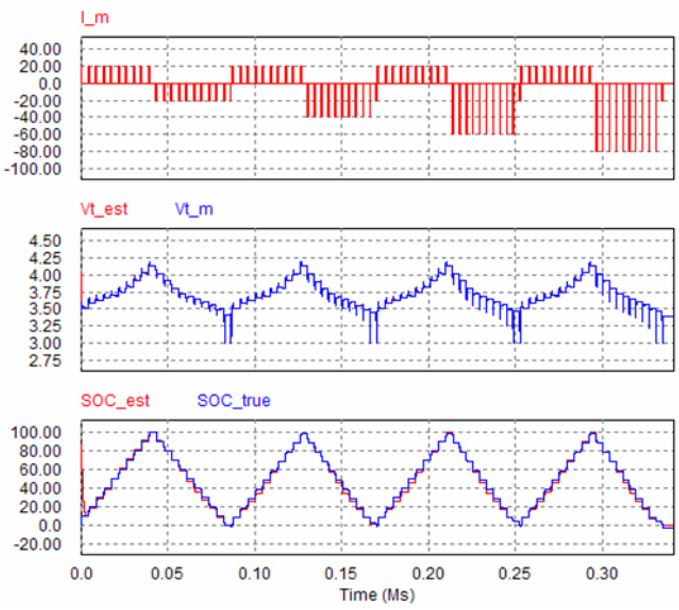

Fig. 19. Experimental data and estimation value from EKF with $0.5 \mathrm{C}$ charging rates and various discharging rates. 


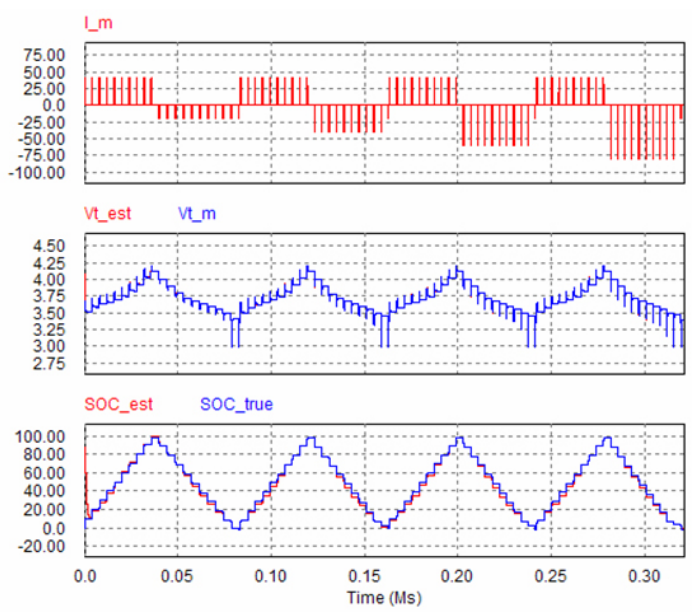

Fig. 20. Experimental data and estimation value from EKF with $1 \mathrm{C}$ charging rates and various discharging rates.

model is not accurate. Therefore, EKF is developed based on an electrical model with high accuracy. Even though EKF adopts a high accuracy model, it may not perform well the estimation. To avoid it, a single dominant varying parameter method is proposed. Considering that the bulk capacitance voltage influences dominantly the battery voltage, it is chosen for varying parameter by following the SOC value. The time varying parameter is implemented with the piecewise continuous linear look-up table. By applying varying parameter, the error of estimation can be reduced up to maximum 5\% SOC over the SOC region and various current magnitudes.

The proposed method is verified through the test of full range of SOC and various current rates. Therefore, the application of the proposed method can be used for the energy storage application which involves the wide range of SOC such as PV power source and the dynamic operation such as wind turbine. It can be expanded into other energy storages. However the dominant parameter should be chosen wisely to keep estimation works well.

\section{REFERENCES}

[1] B. Yang, Y. Makarov, J. Desteese, V. Viswanathan, P. Nyeng, B McManus, and J. Pease, "On the use of energy storage technologies for regulation services in electric power systems with significant penetration of wind energy," Proc. of 5th International Conference on European Electricity Market (EEM), pp. 1-6, 2008.

[2] R. Muhida, A. Mostavan, W. Sujatmiko, M. Park and K. Matsuura, "The 10 years operation of a PV-micro-hydro hybrid system in Taratak, Indonesia," J. of Solar Energy Materials and Solar Cells, Vol. 67, No. 1-4, pp. 621-627, Mar. 2001.

[3] C. N. Rasmussen, "Improving wind power quality with energy storage," Proc. of IEEE PES/IAS Conference on Sustainable Alternative Energy (SAE), pp. 1-7, 2009.

[4] H. Gao, Y. Gao, and M.Ehsani, "A neural network based SRM drive control strategy for regenerative braking in EV and HEV," Proc. of IEEE International Electric Machines and Drives Conference (IEMDC), pp. 571-575, 2001.

[5] J. Chatzakis, K. Kalaitzakis, N. C. Voulgaris, and S .N. Manias, "Designing a new generalized battery management system," IEEE Trans. Ind. Electron., Vol. 50 , No. 5, pp. 990-999, Oct. 2003.

[6] B. S. Bhangu, P. Bentley, D. A. Stone, and C. M. Bingham, "Nonlinear observers for predicting state-of-charge and state-of-health of lead-acid batteries for hybrid-electric vehicles," IEEE Trans. Vehic. Technol., Vol. 54, No. 3, pp. 783-794, May 2005.
[7] A. Vasebi, S. M. T. Bathaee, and M. Partovibakhsh, "Predicting state of charge of lead-acid batteries for hybrid electric vehicles by extended Kalman filter," J. of Energy Conversion and Management, No. 49, pp. 75-82, Jan. 2008.

[8] V. Pop, H. J. Bergveld, J. H. G. Op het Veld, P.P.L. Regtien, D. Danilov, and P. H. L. Notten, "Modeling battery behavior for accurate state-ofcharge indication," J. of Electrochem. Soc., Vol. 153, No. 11, pp. A2013A2022, 2006

[9] W. B. Gu and C. Y. Wang, "Thermal-electrochemical modeling of battery systems," J. Electrochem. Soc., Vol. 147, No. 8, pp. 2910-2922, 2000.

[10] A. Szumanowski and Y. Chang, "Battery Management System Based on Battery Nonlinear Dynamics Modeling," IEEE Trans. Vehic. Technol., Vol. 57, No. 3, pp. 1425-1432, May 2008.

[11] N. A. Windarko, J. Choi, and G. B. Chung, "Improvement of electrical modeling of nimh battery for application of microgrid system," Proc. of IEEE ECCE 2010, pp. 4243-4248, 2010.

[12] J. Im, D. Lim, N. Ayub W., J. Choi, and G. B. Chung, "Electrical modeling of lithium-polymer battery," Trans. of Korean Institute of Power Electronics, Vol. 16, No. 2, Apr. 2010.

[13] S. Piller, M. Perrin and A. Jossen, "Methods for state-of-charge determination and their applications," J. of Power Sources, Vol. 96, pp. 113-120, Jun. 2001.

[14] H. Dai, X. Wei, and Z. Sun, "Online soc estimation of high-power lithium-ion batteries used on hevs," Proc. of IEEE ICVES 2006, pp. $342-347,2006$.

[15] B. S. Bhangu, P. Bentley, D. A. Stone, and C. M. Bingham, "Observer techniques for estimating the state-of-charge and state-of-health of VRLABs for hybrid electric vehicles," Proc. of IEEE Conference VPP, pp. 780-789, 2005

[16] J. Lee, O. Nam, and B. H. Cho, "Li-ion battery SOC estimation method based on the reduced order extended Kalman filtering," J. of Power Sources, Vol. 174, pp. 9-15, 2007.

[17] G. Plett, "Dual and joint ekf for simultaneous soc and soh estimation," in CD-ROM Proc. $21^{\text {st }}$ Electric Vehicle Symposium (EVS21), Apr. 2005.

[18] I.-S. Kim, "A technique for estimating the state of health of lithium batteries through a dual-sliding-mode observer," IEEE Trans. Power Electron., Vol. 23, No. 4, pp. 2027-2034, 2008.

[19] S. Abu-Sharkh, and D. Doerffel, "Rapid test and non-linear model characterisation of solid-state lithium-ion batteries," J. Power Sources, Vol. 130, pp. 266-274, 2004.

[20] V. Pop, H. J. Bergveld, J. H. G. Ophet Veld, P. P. L. Regtien, D. Danilov, and P. H. L. Notten, "Modeling battery behavior for accurate state-ofcharge indication," J. of Electrochem. Soc., Vol. 153, No. 11, p. A2013A2022, 2006.

[21] G. Plett, "Kalman-filter soc estimation for lipb hev cells," in CD-ROM Proc. of EVS19, 2002.

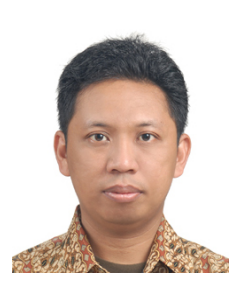

Novie Ayub Windarko received his B.E and M.E in Electrical Engineering from Sepuluh Nopember Institute of Technology, Surabaya Indonesia, in 1999 and 2006 respectively. He received his $\mathrm{Ph}$. D. from School of Electrical Engineering, Chungbuk National University, Korea in 2011. He has been with the Electronic Engineering Polytechnic Institute of Surabaya, Indonesia, since 2000. He was a Visiting Researcher in Tokyo Institute of Technology, Japan, from 2002 to 2003. His research interests are in power electronics, power quality, energy storage systems and renewable energy.

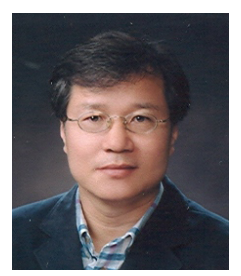

Jaeho Choi received his B.S., M.S., and Ph.D. degrees in electrical engineering from the Seoul National University, Seoul, Korea in 1979, 1981, and 1989, respectively. From 1981 to 1983, he was with the Jungkyoung Technical College, Daejeon, Korea, as a Full-time Lecturer. Since 1983, he has been with the School of Electrical Engineering, Chungbuk National University in Cheongju, Korea, where he is currently a professor. In 1993, 1998, 2003, and 2009, he was a visiting professor at the University of Toronto in Canada each for one year and he was a Danfoss Visiting Professor at the Aalborg University in Denmark in 2000. His research areas include power electronics, power quality problems and solutions, energy storage systems, and renewable energy and microgrid systems. He is an active member of KIEE, KIPE, and IEEE, and he is currently the Editor-in-Chief of JPE. 